\title{
Robust Channel Estimation in Multiuser Downlink 5G Systems Under Channel Uncertainties
}

\author{
Azadeh Pourkabirian and Mohammad Hossein Anisi, Senior Member, IEEE
}

\begin{abstract}
In wireless communication, the performance of the network highly relies on the accuracy of channel state information (CSI). On the other hand, the channel statistics are usually unknown, and the measurement information is lost due to the fading phenomenon. Therefore, we propose a channel estimation approach for downlink communication under channel uncertainty. We apply the Tobit Kalman filter (TKF) method to estimate the hidden state vectors of wireless channels. To minimize the maximum estimation error, a robust minimax minimum estimation error (MSE) estimation approach is developed while the QoS requirements of wireless users is taken into account. We then formulate the minimax problem as a non-cooperative game to find an optimal filter and adjust the best behavior for the worst-case channel uncertainty. We also investigate a scenario in which the actual operating point is not exactly known under model uncertainty. Finally, we investigate the existence and characterization of a saddle point as the solution of the game. Theoretical analysis verifies that our work is robust against the uncertainty of the channel statistics and able to track the true values of the channel states. Additionally, simulation results demonstrate the superiority of the model in terms of MSE value over related techniques.
\end{abstract}

Index Terms - Channel estimation, game theory, minimax optimization, Quality of Service guarantees, $5 \mathrm{G}$ networks.

\section{INTRODUCTION}

$\mathrm{R}$ ECENTLY, the growing number of connected devices has led to rapidly increasing data traffic for next-generation wireless networks. Internet of Things (IoT) [1]-[5] is a new paradigm which connects billions of smart devices to the Internet. However, driven by the vast number of connected devices and massive traffic volumes, IoT will face a major challenge on higher capacity and more spectrum resource. fifth generation (5G) [6]-[10] is considered an efficient solution to provide a manifold increase in channel capacity and introduce new radio enhancements including coordinate multi-point transmission (CoMP), non-orthogonal multiple access (NOMA), millimeter wave (mmWave) communications and multiple-input multiple-output (MIMO) for new service requirements in IoT. However, the heterogeneous IoT devices with various resources and services such as smart home, smart car, smart healthcare, etc., require different types of QoS requirements. To provide QoS, the minimum required signalto-interference-plus-noise ratio (SINR) should be satisfied for these devices. On the other hand, the SINR-constraint satisfaction highly relies on the accurate CSI estimation. In harsh propagation IoT environments where the wireless channel conditions vary, an accurate knowledge of the channel (i.e. perfect CSI) is unavailable. Imperfect CSI significantly

A. Pourkabirian is with the Department of Computer and Information Technology Engineering, Qazvin Branch, Azad University, Qazvin, CO 34199-15195 Iran (e-mail: pourkabirian@qiau.ac.ir).

M. H. Anisi is with the School of Computer Science and Electronic Engineering, University of Essex, Colchester CO4 3SQ, United Kingdom (email:m.anisi@essex.ac.uk). decrease the network performance in terms of the network throughput, and QoS guarantees. Therefore, in absence of perfect CSI, accurate CSI required to be estimated. Channel estimation methods which help obtain accurate CSI, play a key role in spectrum efficiency, QoS guarantee and achieving the maximum capacity of wireless channels.

\section{A. Related Work}

Many channel estimation strategies proposed in the literature [11]-[16]. The works in [17]-[25] investigated the radio resource management assuming channel state information is perfectly known between the transmitter and all the users. Nevertheless, the perfect CSI assumption at all user terminals is unrealistic in practical communications scenarios due to time varying wireless channel conditions, channel estimation errors and quantization errors. In more practical scenarios, only statistical CSI is reasonable and available. Motivated by this challenge, channel estimation techniques [26]-[51] have developed in the literature for wireless communications. In [26], the authors have shown a virtual resource allocation approach taking into consideration inaccurate channel estimation and measurement for device-to-device communications. Authors in [27] presented a user scheduling mechanism for multiple-input multiple-output (MIMO) transmission with active CSI feedback. The aforementioned works [26], [27] obtain CSI via downlink training and channel 
state feedback; thus, they suffer from transmission errors and feedback delay which can substantially decrease the sum capacity. These three impairments can cause a mismatch between the observed and the real CSI. Study [28] claimed that delayed CSI is still useful in channel tracking. It showed that the CSI feedback delay might be less than the channel coherence time considering the user mobility. Authors in [29][33] proposed robust power allocation and resource optimization approaches for heterogeneous wireless networks under channel uncertainties. They transformed these optimization problems into the convex optimization problems which is solved by using Lagrange dual theory and sub gradient algorithm. There have been studies [34]-[37] about the worstcase resource allocation in power domain non-orthogonal multiple access systems by considering uncertainty of CSI. Authors in [38] investigated on IoT traffic with limited frequency resources to provide robust wireless networks and satisfy users' demand. An optimal investment and pricing decisions was presented for cognitive mobile network under spectrum supply uncertainty in [39]. They modeled the interactions between the operator and the users by a Stackelberg game to obtain maximum transmission power levels and channel gains for all users. In [40] authors claimed the only way to measure the CSI from a given node is by receiving a packet containing training symbols from that node within a communication range. To the best of our knowledge, these studies estimate the channel using the known training sequence. This could result in huge amount of the CSI feedback, weak synchronization between transmitter and receiver, capacity limitation and latency-constraint are some of the challenges with these approaches. There are also some channel estimation schemes in [41]-[44] based on the known training sequence. Additionally, Maximum likelihood (ML) channel estimationbased methods were investigated in [45]. The methods depend on the distribution of the noise in which ML solution can be obtained by maximizing a quantity related to the log-likelihood. However, the ML approach use knowledge to improve channel estimation when the noise is not white, and its covariance matrix is known. Authors in [46], [47] developed a channel estimation approach based on maximum a posteriori (MAP) that treats the channel coefficients as random variables and maximized the likelihood of the channel coefficients given the received data.

However, all the above approaches have considered timeinvariant channels meaning that the channel response over a time slot was approximately constant. This assumption is not so practical for IoT networks because of the mobility of wireless nodes, or the scattering objects in wireless mobile communication. The time evolution of the channel must be characterized for a time-varying channel. In this regard, channel tracking approaches [48], [49] were developed to study how the channel changes over time. Authors focused on increasing the received SINR during channel tracking by proposing a precoding and combining design method. In addition, authors in [50] applied a Markov process and the classical Kalman filter (KF) to track the MIMO channel. However, none of the aforementioned works studied censored measurements in channel tracking and estimation whereas censored measurement is one of the most important encountered measurement in wireless systems. The censoring phenomenon is common in $5 \mathrm{G}$ communication and it occurs when the measurement falls outside the range of a value which could result in underestimation or overestimation. Thus, it is vital to model censored measurements in channel estimation process in order to obtain more accurate estimation results. TKF [51]-[56] as an adjusted version of the Kalman Filter is a good choice when our observations are suffering from censorship. Although some studies exist for channel estimation, none of the work has taken the censored measurements into consideration. The major difference between our proposed approach and other prior works is characterizing censored measurements in channel estimation process. In fact, the TKF estimates the channel matrix based on a series of observations under censored and fading measurements. Moreover, channel estimation is one of the complex optimization problems. In this regard, game theory [57] as a powerful mathematical tool, is a good choice for the complex analysis. In multiuser $5 \mathrm{G}$ systems, selfish users compete with each other to obtain more channel capacity in order to fulfill their quality of service requirements. Game theory analyzes the conflict and strategic behavior of wireless users and models the interaction among them. This interaction reflect relation between the user valuation in terms of achievable channel capacity and channel uncertainty. Optimization methods cannot model interactions among wireless users in competitive scenarios. Some works in the literature address the radio resource allocation [58]-[64] and channel estimation [65], [66] problem using game theory. However, among these very few studies, to the best of our knowledge, the proposed approach is the first work that finds an optimal strategy for each user in terms of optimal channel filter to obtain accurate estimation of channel under the worstcase channel uncertainty scenario. The optimal filter gives the best behavior of users under the worst channel conditions. Thus, the proposed game model optimizes the worst-case network performance.

\section{B. Contributions}

In this work, a channel estimation approach is proposed for downlink communication in $5 \mathrm{G}$ networks. The main objective is to optimize the worst-case network performance. In summary, the main contributions of this work are summarized as follows:

- We propose a robust channel assignment approach under channel uncertainty. To enhance channel assignment, CSI needs to be available to adapt the transmissions with the true channel conditions. Therefore, we propose a channel estimation algorithm under the worst channel uncertainty considering that CSI is usually unknown in wireless systems. In practical systems, the measurements are correlated and censored due to the fading phenomenon. Thus, it is vital to model censored measurements in channel estimation process. Therefore, we take both censored and fading measurements in channel estimation into account. We apply the TKF to characterize censored measurements 
in channel estimation. TKF estimates the channel matrix based on a series of observations in noisy and fading environments.

- In the absence of an exact information of the channel, a robust method is required to be less sensitive against imperfect CSI knowledge and obtains low estimation error rates. We develop a robust minimax MSE estimation approach to reduce the channel estimation error. Our robust method optimizes the worst-case performance through minimizing the maximum estimation error.

- Different from existing works, we also use game theory to model strategic behavior of wireless users in channel assignment process for multiuser $5 \mathrm{G}$ systems. In the proposed game model, filters are the strategies and the elements of the uncertainty set are considered to the operating points. Each user chooses a filter as a strategy to estimate channel conditions. According to its estimation, after the user selects a channel, received payoff in terms of allocated capacity is observed. Wireless users compete with each other over wireless channels to obtain higher payoff. The payoff of each user depends on its chosen strategy and the uncertain quantities of the model. Different from previous works, we take into consideration a scenario in which the actual operating point is not exactly known under uncertainty model. Game theory helps find an optimal strategy for each user results in obtaining accurate estimation of channel and increasing users' payoff. The more users' payoff increases, the more users' QoS requirement guarantees. This distributed mathematical framework produces less communication cost than the conventional schemes therefore, it is efficient for large multiuser systems like $5 \mathrm{G}$.

- Finally, we analyze the existence and characterization of a saddle point as the solution of game. We then develop an iterative algorithm to find the saddle point solution of the game. Therefore, we achieve the global solution of the problem. We carry out simulation experiments to validate the performance of our proposed algorithm. Simulation results verify theoretical performance evaluation and ensure the correctness and the effectiveness of our approach in terms of low estimation error and high data rate.

The remainder of the paper is organized as follows: Section II describes the system and uncertainty models. Then the original system is transformed to a state-space system model and formulated by the TKF model for both censored and fading measurements. In Section III, we develop a minimax approach to optimize the worst-case performance of the problem. A game-theoretic formulation of the problem is presented in order to find the optimal filter under worst operating points in Section IV. We then investigate the existence and characterization of a saddle point to obtain the minimax solution. In Section V, we design a new distributed algorithm for the robust user association optimization problem and also provide analytic discussions on the computational complexity. Section VI evaluates the performance of the proposed approach. Finally, in the last section, we conclude the paper in Section VII.

\section{SYSTEM DESCRIPTION}

\section{A. System Model}

We model the downlink transmission of the multiuser cellular communication system including $B$ base stations (BSs), each equipped with $N_{T}$ antennas, and $K$ users, each with one receive antenna. BSs are positioned based on a homogeneous Poisson point process (PPP) $\Phi_{j}$, with intensity $\lambda_{j}$, in the Euclidean plane, i.e. $\Phi_{j} \sim \operatorname{PPP}(\lambda \mathrm{j})$. We also assume that the distribution of wireless users follows a homogeneous PPP $\Phi_{U}$, with intensity $\lambda_{U}$, which is independent of $\Phi_{j}$ for all $j \in$ $\{1, \ldots, J\}$. Each user chooses a BS that fulfills its QoS requirements and change its chosen BS if necessary. According to Shannon's formula, the received SINR of user $k$ from a typical BS can be given as below:

$$
\gamma_{k}=\frac{P_{k}\left|h_{k} g_{k}\right|^{2}}{\sum_{i \neq k}\left(P_{i}\left|h_{k} g_{k}\right|^{2}\right)+\sigma_{k}^{2}}
$$

where $P_{k}$ indicates the transmit power of a typical BS to user $k$, $g_{k}$ identifies the user $k$ 's equalizing gain, $h_{k} \in H_{k}, H_{k} \in$ $\mathbb{C}^{N_{T} \times 1}$ is the random fading coefficient from the BS to the user $k, \sum_{i \neq k}\left(P_{i}\left|h_{k} g_{k}\right|^{2}\right)$ indicates the aggregate interference from all other interfering users, and $\sigma_{k}^{2}$ is the variance of the noise. The true channel can be expressed as $H_{k}=\widehat{H}_{k}+e_{k}$, where $\widehat{H}_{k}$ is the estimated channel coefficient (vector) and $e_{k}$ denotes the channel estimation error whereas $e_{k} \sim \mathcal{C N}\left(0, \xi_{k}^{2} I\right)$ in which $\xi_{k}^{2}$ is the error variance and it relies on the channel estimation quality.

Due to the dramatically increasing demand for the IoT services, wireless users always look for a serving BS to satisfy their QoS requirements. The QoS requirement for a user can be guaranteed when the received SINR of the user is higher than a certain threshold (i.e., $\gamma_{k}>\gamma_{\min }$ ). With imperfect CSI, a user can no longer make sure that a serving BS fulfills the SINR requirement. Therefore, an estimation method is required to obtain the accurate channel information over the whole range of uncertain conditions. In the subsequent section, we apply the Tobit Kalman filter to estimate the channel information considering fading and censored measurements.

\section{B. State-Space Model Under Channel Uncertainty}

In this section, we transform the communication system model to a state-space model description as follows:

$$
\mathrm{X}_{t}=F_{t} X_{t-1}+C_{t} W_{t}
$$

where $X_{t} \in \mathbb{R}^{K \times 1}$ denotes the state vector at time $t, F_{t} \in$ $\mathbb{R}^{K \times K}$ presents the state transition matrix, $X_{t-1}$ identifies the previous state vector at time $t-1, C_{t} \in \mathbb{R}^{K \times N_{T}}$ is timevarying matrix with appropriate dimension, $W_{t} \in \mathbb{R}^{N_{T} \times 1}$ indicates Gaussian observation noise vector with covariance matrix $Q_{t}, W_{t} \sim \mathcal{N}\left(0, Q_{t}\right)$ and $t$ stands for the discrete time step. Thus, in a system with fading measurements under the Rician fading model we have

$$
Z_{t}=H_{t} X_{t}+V_{t}
$$


where $Z_{t}$ is the measurement vector, $H_{t}$ denotes the matrix of random fading coefficients which describe a wireless channel and $V_{t}$ is the white Gaussian noise vector with covariance matrix $R_{t}$.

Assumption 1. The channel coefficients at time step $t$ are identified as $H_{t}=\operatorname{diag}\left\{H_{t, 1}, H_{t, 2}, \ldots, H_{t, K}\right\}$ where $H_{t, k}=$ $\left[h_{k}^{1}, h_{k}^{2}, \ldots, h_{k}^{N_{T}}\right]^{\prime}$ denotes the coefficients vector of user $k$ and $h_{k}^{n}$ is the random variable with the mean $\bar{h}_{k}^{n}$ and the variance $\tilde{h}_{k}^{n}$ which regulates the fading phenomenon between the $n^{\text {th }}$ antenna and $k^{t h}$ user. For the sake of simplicity, we use $H_{k}$ instead of $H_{t, k}$ and $h_{k}$ for $h_{k}^{i}$ in the rest of this paper.

\section{Tobit Kalman Filter Formulation}

Now, we model the channel estimation problem as the Tobit Kalman Filter [52] that is the effective channel estimation method to calculate both censored and fading measurements under high channel uncertainty. The TKF estimates the unknown channel parameters based on a series of observations (i.e., signal measurements) over time.

$$
\begin{aligned}
Z_{t}^{*} & =H_{t} X_{t}+V_{t} \\
Z_{t} & =\left\{\begin{array}{lr}
Z_{t}^{*}, & a<Z_{t}^{*}<b \\
a, & Z_{t}^{*} \leq a \\
b, & Z_{t}^{*} \geq b
\end{array}\right.
\end{aligned}
$$

where $Z_{t} \in \mathbb{R}^{K \times 1}$ denotes the censored measurement vector, $Z_{t}^{*}$ is considered as the latent variable and $\mathrm{a}, \mathrm{b}$ are the lower and the upper thresholds of the uncensored region, respectively. For the sake of simplicity, we assume $\{a, b\}=\tau$. Thus, (5) can be written as follows:

$$
Z_{t}=q_{t} Z_{t}^{*}+\left(I-q_{t}\right) \tau
$$

where $q_{t}$ regulates the censoring phenomena meaning that whether $Z_{t}$ is censored, $q_{t}=0$; or not, $q_{t}=1$. Notably, if no censoring occurs, the value of observation is $Z_{t}=Z_{t}^{*}$, otherwise the output measurement is $Z_{t}=\tau$.

Remark 1. We define the censored measurement vector as $Z_{t}=\left[z_{t, 1}, z_{t, 2}, \ldots, z_{t, K}\right]^{\prime}$ and the latent observations vector as $Z_{t}^{*}=\left[z_{t, 1}^{*}, z_{t, 2}^{*}, \ldots, z_{t, K}^{*}\right]^{\prime}$ for all users at time step $t$. As is well known, the censored measurement $Z_{t}$ does not rely on the state vector $X_{t}$, when the latent observations $Z_{t}^{*}$ is outside the uncensored region. Therefore, we extend (5) as follows:

$$
z_{t, k}=\left\{\begin{array}{lr}
z_{t, k}^{*}, & a<z_{t, k}^{*}<b \\
a, & z_{t, k}^{*} \leq a \\
b, & z_{t, k}^{*} \geq b
\end{array} \quad k=1,2, \ldots, K\right.
$$

The TKF is typically divided into two distinct phases: the predict stage and the update stage. In the predict stage, TKF estimates the current state using the previous the state estimate. Then, in the update phase, the algorithm combines this prediction with current measured information to refine the state estimate. The process is repeated each time and a new state estimate is produced through the predicted and measured state.
The predict and update functions of TKF are defined as follows:

\section{1) The Predict Stage:}

We calculate a posteriori estimation $X_{t}$ that is a linear combination of the priori estimation $X_{t-1}$.

$$
X_{t \mid t-1}=F_{t-1} X_{t-1 \mid t-1}
$$

where $X_{t \mid t-1}$ denotes a posteriori state estimate and $X_{t-1 \mid t-1}$ is a priori state estimate. Therefore, the error covariance of the priori and posteriori state estimates up to time $t-1$ can be written as:

$$
\operatorname{cov}_{t \mid t-1}=F_{t-1} \operatorname{var}_{t-1 \mid t-1} F_{t-1}^{T}+Q_{t-1}
$$

where $\operatorname{var}_{t-1 \mid t-1}=\operatorname{var}\left(X_{t-1 \mid t-1}\right)$ denotes the previous a posteriori state estimate of error variance.

\section{2) The Update Stage:}

The state error covariance will be decreased in the update stage because the state estimates are updated with current measurements in this stage considering measurement errors. Therefore, the current estimate based on all observations up to time $t$ can be expressed as:

$$
X_{t \mid t}=X_{t \mid t-1}+K_{t}\left(Z_{t}-\mathbb{E}\left(Z_{t} \mid X_{t \mid t-1}\right)\right)
$$

where $\mathbb{E}\left(Z_{t}\right)$ stands for the expected value of the measurements including censored and uncensored measurements and it has been calculated in [54], and $K_{t}$ denotes the Kalman Gain that is defined as:

$$
K_{t}=R_{t, 1} R_{t, 2}^{-1}
$$

where the measurement noise covariance matrices $R_{1}$ and $R_{2}$ are described in detail in [67] and take the form

$$
\begin{aligned}
R_{t, 1} & =\operatorname{cov}_{t \mid t-1} H_{t}^{T} P_{t, u n} \\
R_{t, 2} & =P_{t, u n} H_{t} \operatorname{cov}_{t \mid t-1} H_{t}^{T} P_{t, u n}+R_{t}^{*}
\end{aligned}
$$

where $\operatorname{cov}_{t \mid t-1}=\mathbb{E}\left(\left(X_{t}-X_{t \mid t-1}\right)\left(X_{t}-X_{t \mid t-1}\right)^{T}\right)$ denotes the covariance of the state estimate, $R_{t}^{*}$ identifies a diagonal matrix that includes the truncated variances of $z_{t, k}^{*}$ for $k=$ $1, \ldots, n_{u n} . n_{u n} \leq K$, and $P_{t, u n}$ is a diagonal matrix that stands for the probabilities of a measurement to be uncensored and it is calculated as follows [65]:

$$
P_{t, u n}=\operatorname{diag}\left[\Phi\left(b_{t, 1}\right)-\Phi\left(a_{t, 1}\right) \quad \ldots \quad \Phi\left(b_{t, K}\right)-\Phi\left(a_{t, K}\right)\right]^{\prime}
$$

As it is clear, the $k^{t h}$ element of diagonal matrix $P_{t, u n}$, determines the probability that a latent measurement $z_{t, k}^{*}$ belongs to the uncensored region and $\Phi$ is the cumulative 
distribution function of $\mathrm{N}(0,1)$. So, the covariance update equation of the measurement can be written as:

$$
\operatorname{cov}_{t \mid t}=\left(I-K_{t} H_{t} P_{t, u n}\right) \cdot \operatorname{cov}_{t \mid t-1}
$$

where $I$ denotes the identity matrix.

Remark 2. As mentioned before, the indicator $q_{t, k}$ regulates the censoring phenomena of $z_{t, k}$ where $q_{t}=$ $\operatorname{diag}\left\{q_{t, 1}, q_{t, 2}, \ldots, q_{t, K}\right\}$ identifies censoring phenomena for all users at time step $t$. More specifically, $q_{t, k}=$ $\left[q_{t, k}^{1}, q_{t, k}^{2}, \ldots, q_{t, k}^{N_{T}}\right]^{\prime}$, in which $q_{t, k}^{i}$ determines censoring phenomena in $i^{\text {th }}$ channel for the user $k$ at time step $t$. Thus, the censoring probability $P_{t, k}^{i, c}$ can be written as:

$$
\operatorname{Prob}\left\{q_{t, k}^{i}=1\right\}=P_{t, k}^{i, u n}
$$

and

$$
\operatorname{Prob}\left\{q_{t, k}^{i}=0\right\}=P_{t, k}^{i, c}
$$

where $P_{t, k}^{i, u n}$ denotes the probability that no censoring occurs. For simplicity, we consider $P_{k, u n}$ instead of $P_{t, k}^{i, u n}$ and $P_{k, c}$ for $P_{t, k}^{i, c}$ in the rest of this paper. Now, we calculate the censoring probability that is given by [68]:

$$
P_{k, c} \approx \Phi\left(\frac{\left(\sum_{d=0}^{l_{t}} \bar{H}_{t, k}(d) x_{t-d \mid t-d-1}\right)-\tau}{\sqrt{R_{t}}}\right)
$$

where $\bar{H}_{t, k}(d)$ determines the mean of $H_{t, k}(d)=$ $\left[h_{k}^{1}(d), h_{k}^{2}(d), \ldots, h_{k}^{N_{T}}(d)\right]^{\prime}$ which regulates the fading phenomena in the $i^{t h}$ channel for the user $k$, and $x_{t-d \mid t-d-1}$ states the one- step prediction of $x_{t-d}$, in which $d=0,1, \ldots, l_{t}$ model $L$ th-order Rician fading channel, $l_{t}=\min \{L, t\}$ where $L$ is the order of the $L$ th-order Rician fading [69]. Next, we show analytically the one-step measurement prediction based on the TKF with both censored and fading measurements through the following theorem.

Theorem 1. The one-step measurement prediction based on the TKF under both censored and fading measurements is expressed as:

$\mathbb{E}\left\{z_{t, k} \mid X_{t \mid t-1}\right\}=P_{k, u n}\left[X_{t \mid t-1}+\lambda\left(\frac{\tau-X_{t \mid t-1}}{\sqrt{R_{t}}}\right) \mathcal{R}_{t}\right]+\left(P_{k, C}\right) \tau$

where $\lambda(\alpha)=\frac{\phi(\alpha)}{1-\Phi(\alpha)}$

In which $\phi(\alpha)$ is the probability density function (PDF) and $\Phi(\alpha)$ denotes the cumulative distribution function (CDF) of a Gaussian random variable that are derived as below:

and

$$
\phi\left(\frac{\tau-X_{t \mid t-1}}{\sqrt{R_{t}}}\right)=\frac{1}{\sqrt{2 \pi}} e^{-\frac{\left(\tau-X_{t \mid t-1}\right)^{2}}{2 R_{t}}}
$$

$$
\Phi\left(\frac{\tau-X_{t \mid t-1}}{\sqrt{R_{t}}}\right)=\int_{-\infty}^{\tau} \frac{1}{\sqrt{2 \pi R_{t}}} e^{-\frac{\left(z_{t, k}-X_{t \mid t-1}\right)^{2}}{2 R_{t}}} d z_{t, k}
$$

Therefore,

$$
\lambda\left(\frac{\tau-X_{t \mid t-1}}{\sqrt{R_{t}}}\right) \mathcal{R}_{t}=\operatorname{diag}\left\{\lambda\left(\frac{\tau_{k}-x_{t \mid t-1, k}}{\sqrt{R_{t}^{k, k}}}\right)\right\}
$$

where $\mathcal{R}_{t}=\left[\sqrt{R_{t}^{1,1}}, \ldots, \sqrt{R_{t}^{k, k}}\right]$ in [52].

\section{Proof. See Appendix A.}

However, the above estimations may not be optimal due to the channel estimation error as follows:

$$
h_{k}=\hat{h}_{k}+e_{k}
$$

To obtain a more accurate estimate, we need to minimize the estimation error.

\section{QOS-CONSTRAINT CHANNEL ASSIGNMENT PROBLEM}

In a downlink communication, wireless users are constantly looking for a BS that fulfills their QoS requirements. However, under the channel uncertainty and with channel estimation error, the expected rate of each user may be not guaranteed. Therefore, the BS must exceed the average transmission performance more than a target value to ensure that the required rate of each user is satisfied. To design a QoS- constraint scheme, we consider the worst-case SINR requirement of each user under channel uncertainty. Our objective is to maximize the received SINR for each user subject to predefined user's QoS target. In this regard, we formulate the QoS-constraint problem as:

$$
\begin{aligned}
& \max _{P, g} \sum_{k=1}^{K} \frac{P_{k}\left|h_{k} g_{k}\right|^{2}}{\sum_{i \neq k}\left(P_{i}\left|h_{k} g_{k}\right|^{2}\right)+\sigma_{k}^{2}} \\
& \text { s.t. } \quad \operatorname{SINR}_{k}>\gamma_{\text {min }}, \text { for } 1 \leq k \leq K
\end{aligned}
$$

We, then measure the MSE of the estimator to evaluates the estimation performance. The MSE value determines the difference between the true values and the estimated values. Under uncertainty model in which the channel is estimated, the objective is to minimize the size of the channel estimation error in order to all users' QoS requirements are satisfied. For this purpose, we calculate the MSE of the estimation as below:

$$
\begin{aligned}
& M S E_{k}=\sum_{j=1}^{N_{T}}\left|g_{k}\right|^{2} P_{j}^{H}\left(\hat{h}_{k}^{H} \hat{h}_{k}+\sigma_{e}^{2} I\right) P_{j}+\sigma_{n}^{2}\left|g_{k}\right|^{2}- \\
& g_{k} \hat{h}_{k} P_{k}-g_{k} \hat{h}_{k}^{H} P_{k}^{H}+1
\end{aligned}
$$

where $M S E_{k}$ identifies the mean square error of channel estimation for the $k^{\text {th }}$ user, and $f_{k} \in F=\operatorname{diag}\left(f_{1}, f_{2}, \ldots, f_{K}\right)$, denotes the user's filter (i.e., equalization coefficient). On the other hand, the total MSE can be defined as:

$M S E=\sum_{k=1}^{K} M S E_{k}$

To improve the quality of estimation, we develop a minimax MSE approach that minimize the maximum estimation error for all users. As a result, the received SINR of each user is 
maximized. Thus, the QoS-constraint problem in (24) can be written as:

$$
\begin{aligned}
& \min _{G, f_{1}, \ldots, f_{k}} \max _{\left\|e_{k}\right\| \leq \varepsilon_{k}} \sum_{k=1}^{K} M S E_{k} \\
& \text { s.t. } \operatorname{Pr}\left\{\frac{P_{k}\left|h_{k} g_{k}\right|^{2}}{\sum_{i \neq k}\left(P_{i}\left|h_{k} g_{k}\right|^{2}\right)+\sigma_{k}^{2}}<\gamma_{\min }\right\} \leq \rho
\end{aligned}
$$

Where

$$
\begin{aligned}
& M S E_{k}=\left(\left(f_{k}\right)^{-1} \sum_{j=1}^{N_{T}}\left|g_{k}\right|^{2} p_{j}^{H}\left(h_{k}^{H} h_{k}\right) p_{j}\right)-g_{k} h_{k} p_{k}- \\
& g_{k}^{H} h_{k}^{H} p_{k}^{H}+\left(f_{k}\right)^{-1}\left|g_{k}\right|^{2} \sigma_{k}^{2}+1=\left(f_{k}\right)^{-1}\left(\left\|g_{k} h_{k} P-Q_{k}\right\|^{2}+\right. \\
& \left.\left\|g_{k}\right\|^{2} \sigma_{k}^{2}\right)
\end{aligned}
$$

where $\operatorname{tr}\left\{P P^{H}\right\} \leq P^{\text {total }}$ and

$Q_{k}=\left[0_{N_{k} \times \sum_{i=1}^{k-1} N_{i}}, I, 0_{N_{k} \times \sum_{i=k+1}^{K} N_{i}}\right]$.

Each user would self-adjust its optimal filter, over admissible channel $h_{k}$ to minimize the worst-case MSE. The optimal filter is the best policy under the worst-case uncertainty that leads minimum estimation error. The filter of user $k, f_{k}$ can be defined as:

$f_{k}=\frac{\left(g_{k} h_{k}\right)^{H}}{g h_{k}\left(g h_{k}\right)^{H}+\sigma_{k}^{2}}$

Notably, we assume that each BS optimizes its filter G under the worst-case scenario, then the user $k$ would estimate the channel coefficient $H_{k} G$, afterward the user adjusts its filter. Therefore, considering the fixed filter $\mathrm{G}$, the QoS target will be fulfilled for each user throughout the MSE solution.

Lemma 1. The original minimax optimization problem in (27) can be rewritten as the following convex problem:

$\min _{\left\{Q_{k} \in \mathbb{C}^{\left.N_{T} \times N_{T}\right\}}\right.} \sum_{k=1}^{K} \operatorname{Tr}\left(Q_{k}\right)$

s.t. $Q_{k} \geq 0$

proof. The channel uncertainty set of the user $k$ is given as:

$H_{k}=\widehat{H}_{k}+e_{k}, \quad\left\|e_{k}\right\|_{2}=\left\|H_{k}-\widehat{H}_{k}\right\|_{2} \leq \varepsilon$

Based on the S-procedure [70], we convert the non-convex constraint into linear matrix inequality (LMI) form. Therefore, we have

$e_{i}^{H} \mathrm{I} e_{i}-\varepsilon^{2} \leq 0 \Rightarrow e_{i}^{H}\left(\sum_{n \neq k} Q_{n}-Q_{k} / \gamma_{\min }\right) e_{i}+$

$2 \operatorname{Re}\left(\widehat{H}_{i}^{H}\left(\sum_{n=k+1}^{K} Q_{n}-Q_{k} / \gamma_{\min }\right) e_{i}\right)+\widehat{H}_{i}^{H}\left(\sum_{n=k+1}^{K} Q_{n}-\right.$

$\left.Q_{k} / \gamma_{\text {min }}\right) \widehat{H}_{i}+\sigma_{i}^{2} \leq 0$, for $i=k, \ldots, K$.

Now, we can rewrite the constraint (30b) as follow:

$C_{k i}=\left[\begin{array}{cc}\lambda_{k i} \mathrm{I}+\phi_{k}+v_{k} & \phi_{k} \widehat{H} \\ \widehat{H}_{i}^{H} \phi_{k} & \widehat{H}_{i}^{H} \phi_{k} \widehat{H}-\sigma_{k}^{2}-\lambda_{k i} \varepsilon^{2}\end{array}\right] \geq 0$

where $\lambda_{k i} \geq 0$ is a diagonal element of the diagonal matrix , $\phi_{k}=\frac{Q_{k}}{\gamma_{\min }}-\sum_{n=k+1}^{K} Q_{n}$ and $v_{k}=-\sum_{n=1}^{k-1} Q_{n}$.

So, the theorem holds.

Theorem 2. The user's QoS requirement will be guaranteed, for user $k$ (i.e., $\gamma_{k} \geq \gamma_{\min }$ ), if $f_{k}^{o p t}$ is the optimal filter for user $k$ and $\operatorname{prob}\left\{M S E_{k}\left(g_{k}, f_{k}^{o p t}\right)>\epsilon_{k}\right\} \leq \varepsilon, \epsilon_{k} \in(0,1)$,

where $\epsilon_{k}=\frac{1}{1+\gamma_{\min }}, \quad \forall k \in\{1, \ldots, k\}$.

\section{Proof. See Appendix B.}

It is worth noting that, the above claim will be satisfied when one of the filters is fixed in the uncertainty model.

\section{A GAME THEORETICAL MODEL FOR CHANNEL ESTIMATION}

We formulate the channel estimation problem using game theory to study the interaction among wireless users in multiuser 5G systems and present the relation between the channel condition and user valuation. Our proposed distributed game-based approach is run independently at each user equipment (UE). The centralized methods require a central controller to collect, process, and broadcast information over the network results in an increase in communication overhead. Unlike centralized approaches, distributed algorithms like the proposed method only draws upon local information without the need to access the complete network information. In our method, each user estimates the channels coefficients and selects an appropriate channel without any interaction with other users in a distributed manner. It maintains its own selfadaptive channel matrix and updates its value from the SINR information that is periodically sent by the serving BS. Also, there is no need for information exchange over the network. This reduces the number of messages exchanged between various nodes in the network and causes a decrease in signaling overhead.

In the proposed game model, wireless users are the players who select a filter (i.e., equalization coefficient) from their strategy set to maximize payoff under the worst channel uncertainty. The payoff of a player is evaluated in terms of the received SINR by the player. We also assume that the set of filters which a player can adjust are its strategy set. In the presence of channel uncertainty, we consider the elements of the uncertainty set (i.e., channel coefficients) as the operating points of the game. In general, our game model is defined formally as $\mathcal{G}(\mathcal{H}, \mathcal{S}, U)$ where $\mathcal{H} \subset H$ denotes the operating points set, $\mathcal{S} \subset F$ identifies the set of possible strategies where $F=F_{1} \times F_{2} \times \ldots \times F_{K}$, in which $F_{K}=\left\{f_{k}^{1}, f_{k}^{2}, \ldots, f_{k}^{n}\right\}$ is the set of pure strategies of user $k$, and $U: \mathcal{H} \times \mathcal{S} \rightarrow \mathbb{R}$ defines the payoff function which is minimized over $\mathcal{H}$ and maximized over $\mathcal{S}$. More specifically, the payoff function of player $k$, (i.e., $U_{k}$ ) can be defined as below:

$U_{k}\left(f_{k}^{o p t}\left(h_{k}\right), f_{k}\left(h_{k}\right)\right)=R_{k}\left(f_{k}^{o p t}\left(h_{k}\right), f_{k}\left(h_{k}\right)\right)-$

$C_{k}\left(f_{k}^{o p t}\left(h_{k}\right), f_{k}\left(h_{k}\right)\right)$

Where $R_{k}\left(f_{k}^{o p t}\left(h_{k}\right), f_{k}\left(h_{k}\right)\right)=\gamma_{k}$ denotes player $k$ 's revenue in terms of the received SINR, $f_{k}^{\text {opt }}$ states an optimal filter which the user $k$ adjust as best response against the worst channel conditions, and $C_{k}$ is the linear cost function that models censoring and fading and it is calculated using (7). The 
goal is finding an optimal filter which maximizes the user's payoff for a given operating point. Therefore, we can rewrite (35) as follows:

$U\left(f_{k}^{o p t}\left(h_{k}\right), f_{k}\left(h_{k}\right)\right)=U^{o p t}\left(h_{k}\right) \triangleq \sup _{f_{k} \in \mathcal{S}} U\left(f_{k}, h_{k}\right)$

Let $h_{k}^{w}\left(f_{k}\right)$ be as a worst operating point for $f_{k}$, as bellow:

$h_{k}^{w}\left(f_{k}\right) \in \arg \min _{h_{k} \in \mathcal{H}} U\left(f_{k}, h_{k}\right) \triangleq W\left(\mathcal{H}, f_{k}\right)$

By finding optimal filters for every $h_{k}^{w}$ in the uncertainty set $\left(f_{k}^{o p t}, h_{k}^{w}\right)$, we can achieve the optimal worst-case performance. The optimal worst-case performance is the solution of the minimax problem. In other words, the minimax approach minimizes the maximum estimation error that result in optimizing the worst-case performance. Thus, the minimax problem is solved by $\left(f_{k}^{o p t}, h_{k}^{w}\right)$. On the other hand, $\left(f_{k}^{L}, h_{k}^{L}\right)$ is a saddle point solution to the game. As a results, we can achieve the solution of the problem by $\left(f_{k}^{o p t}, h_{k}^{w}\right)$. The objective is to find a robust filter, $f_{k}^{R}$, for the game in order to achieve the optimal worst-case performance. Thus, we define a robust filter, $f_{k}^{R}$, for the game $\mathcal{G}$ as follow:

$f_{k}^{R}=\arg \max _{f_{k} \in \mathcal{S}, h_{k} \in \mathcal{H}} U\left(f_{k}, h_{k}\right)$

When a worst operating point does not exist for the original uncertainty set $\mathcal{H}$, i.e., an extension $h^{w} \subset \mathcal{H}$ can be sough, a least favorable operating point $h_{k}^{L}$ can be defined for the game. $h_{k}^{L}$ that is the duality of the worst operating point, is expressed as bellow:

$h_{k}^{L} \in \arg \min _{h_{k} \in \mathcal{H}} U^{o p t}\left(h_{k}\right)$

Noticing that if $\left(f_{k}^{L}, h_{k}^{L}\right)$ is a saddle point solution to the game then $f_{k}^{L}$ is a minimax robust filter for the problem. That is because if $\left(f_{k}^{L}, h_{k}^{L}\right)$ is a saddle point then $f_{k}^{L}=f_{k}^{o p t}$. Thus, the worst-case performance of the game is attained at $h_{k}^{L}$ and since $f_{k}^{L}$ is the optimal filter, no other filter gives better behavior at $h_{k}^{L}$. Therefore $f_{k}^{L}=f_{k}^{o p t}=f_{k}^{R}$ is the minimax robust filter. In this way, we could find the solution of the minimax problem in (27).

Theorem 3. if $\left(f_{k}^{L}, h_{k}^{L}\right)$ is a saddle point of the game $\mathcal{G}$ then $f_{k}^{L}$ is a minimax robust filter for the game.

Proof. See Appendix C.

\section{THE PROPOSED CHANNEL ASSIGNMENT ALGORITHM}

\section{A. Algorithm Development}

Here, we establish a new iterative algorithm to implement the channel assignment problem and achieve the optimal worstcase performance. The algorithm achieves a saddle point $\left(f_{k}^{R}, h_{k}^{L}\right)$ as the solution of the game under the worst channel uncertainty. In fact, the proposed algorithm is converged to the game equilibrium as the solution of the problem. In the proposed algorithm, each user selects a BS and then adjusts a filter, $f_{k}$, in order to estimate the current state of the channel. The channel estimation is performed based on the priori state estimate and the current observations. A user estimates the worst channel condition given pilot signal. The user then finds a filter which gives the best channel gain in the worst channel conditions. The channel capacity is limited for large values of estimation error. Thus, the user should calculate a robust filter which minimizes the estimation error to achieve the optimal performance under the worst-case channel uncertainty. The user then evaluates its payoff in terms of allocated channel capacity. The payoff of each user depends on the channel estimation quality. The user updates the channel matrix and changes its chosen BS if its QoS requirements are not fulfilled by allocated channel. The pseudo-code of the algorithm is presented in Algorithm 1.

\section{ALGORITHM 1. CHANNEL ASSIGNMENT ALGORITHM}

1. Set $\mathrm{t}=0$;

2. Initialize the state estimate $X_{0}$ for each user

3. For all users

4. Select a $B S$

5. Set $\gamma_{\min }, F_{0}, C_{0}, W_{0}, V_{0}$;

6. For each $B S_{j}$

7. Set $P_{k}^{j}, h_{k}, g_{k}, \sigma_{k}^{2}, \epsilon_{k}$ for user $k$

8. Calculate $g^{\text {opt }}$

9. Set $Q_{k}, H_{t, k}$;

10. Repeat

11. adjust filter $f_{k}$ for user $k$ according to $M S E_{k}\left(g^{o p t}, f_{k}\right) \leq \epsilon_{k}$,

12. Find a worst operating point for $f_{K}$ $h_{k}^{w}\left(f_{k}\right) \in \arg \min _{h_{K} \in \mathcal{H}} U\left(f_{K}, h_{K}\right)$

13. Calculate a minimax robust filter $f_{k}^{R}$ $f_{k}^{R}=\arg \max _{f_{K} \in \mathcal{S}, h_{K} \in \mathcal{H}} U\left(f_{k}, h_{k}^{w}\right)$

14. Observe payoff of each user as $\gamma_{k}$ 15. $\mathrm{t}=\mathrm{t}+1$

16. Until $t \geq t_{\max }$ or $\gamma_{k} \geq \gamma_{\min }$

17. Update $X_{t, k}=F_{t} X_{t-1}+C_{t} W_{t}$ based on all observations up to time $t$

18. Update $Z_{t, k}=H_{t} X_{t}+V_{t}$ with current measurements;

19. End for user loop

\section{B. Complexity Analysis}

In this section, we analyze the computational complexity of the proposed approach. The main complexity of our algorithm is solving the MSE problem (53) that is dominated by calculating (54). The number of arithmetic operations of calculating MSE problem is dominated by computing the number of floating multiplications. According to [71], the complexity of multiplication of two matrices, $A \in \mathbb{C}^{N \times M}$ and $B \in \mathbb{C}^{M \times P}$ is on the order of $O(N M P)$ and the cost of calculating $A^{-1}$ is $O\left(N^{3}\right)$. Furthermore, the matrix determinant has a cost of $O\left(N^{3}\right)$ and the complexity of calculating decomposition of matrix $A$ is on the order of $O\left(N M^{2}\right)$. Therefore, according to the above complexity bases, the total complexity of all $K$ MSE constraints computations is on the order of $O\left(K B N_{T}^{3}\right)$. 
Next, the cost of finding $h_{k}^{w}$ and $f_{k}^{R}$ including sorting and finding $\arg \min$ and $\arg \max$ over a set of $N_{T}$ variables that requires $O\left(N_{T} \log N_{T}\right)$. Based on the stopping criterion of the algorithm, the maximum number of iterations required for the algorithm convergence is between $K$ and $t_{\max } K$ which $t_{\max }$ is a constant factor. Thus, the total complexity of the proposed algorithm is $O\left(K B N_{T}^{4} \log \left(N_{T}\right)\right)$ beside the $O\left(K^{3}\right)$ operations for the initial matrix inversion.

\section{SIMULATION RESULTS}

In this section, we present numerical results to evaluate the performance of the proposed approach which is referred to as "Robust". The performance of the work is compared with the following methods: a robust SINR-constrained symbol-level multiuser precoding design in [72], labeled as SLMP, a robust multiuser MISO transmit optimization scheme in [73], known as RMTO, the non-robust scenario of our approach, referred to as "Non-robust" and the non-robust intelligent reflecting surfaces assisted MISO method in [74], marked as "Non-robust IRS". MATLAB software and the optimization solver CVX [75] are used for simulation and performance evaluation. The following parameter setting are used for simulation settings. We consider a multiuser downlink channel at the transmitter in which each BS is equipped with $N_{T}=6$ antennas and $k=6$ wireless users [72]. Single-antenna users are randomly distributed. The estimate channel vectors $\left\{\widehat{H}_{k}\right\}_{k=1}^{6}$ are randomly generated according to complex Gaussian with zero mean and unit variance. Moreover, the error vectors $\left\{e_{k}\right\}_{k=1}^{6}$ are considered as the zero-mean complex Gaussian distribution where we assume all the users' channels have the same identical error variance $\xi_{k}^{2}=\xi^{2}=0.001$. All experiments are averaged over 1300 channel realizations which each realization consists of 100 channel estimates. The bounds on the uncertainty $\varepsilon=$ 0.1 , the noise variance $\sigma_{k}^{2}=0.01$, and the MSE targets $\epsilon_{k}=$ 0.15 are assumed to be the same for all users. We consider a target SINR $\gamma_{\min }=10 \mathrm{~dB}$ to satisfy different QoS requirements for users and assume the SINR outage probability as $\rho=0.1$ which means that a $90 \%$ SINR satisfaction probability is desired.

In Fig. 1, we plot the users' QoS guarantees in the presence of uncertainties. We examined the performance of each method in term of the average transmitted power versus QoS target under randomly generated 1300 channel realizations with the same error variance $\xi^{2}=0.001$ for all channels. We run the experiments with $k=6$ and $k=3$ wireless users. It is clear from the simulation results that all methods were able to satisfy QoS requirements for all SINR targets less than or equal to 10 $\mathrm{dB}$. The proposed approach finds a worst-case robustness design for a general class of uncertainty sets that guarantees the desired QoS requirements. When the estimation error is increased, the performance of the network gets aggravated. Hence, the proposed approach guarantees appropriate robustness compared to the existing methods under channel uncertainties which leads to higher data rate and more spectral efficiency in the worst channel condition. Therefore, although our channel estimation approach is not dealing with power consumption but considering that our work outperforms other works in terms of estimation accuracy and spectral efficiency, the power consumption is indirectly reduced. Furthermore, for fewer number of users, the network requires less power to guarantee the users' SINR requirements. As a result, the extra power needed to guarantee $\mathrm{QoS}$ becomes lower by decreasing the number of users.

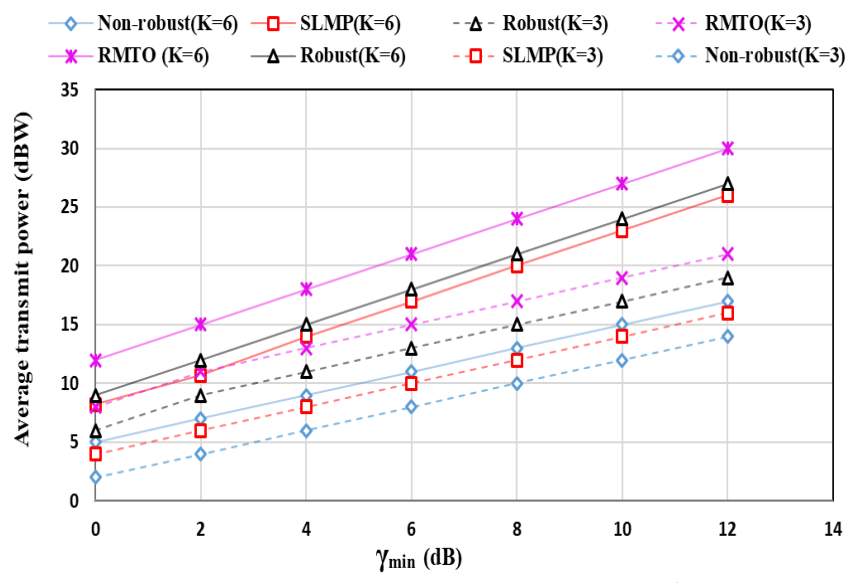

Fig. 1. Transmit power versus QoS guarantees $\left(\gamma_{\min }\right)$ for $\xi^{2}=0.001$, $k=6$ and $k=3$.

Fig. 2 demonstrates the transmit power versus QoS target under larger error variance $\xi^{2}=0.01$ with $k=6$ wireless users. It follows from the figure that as uncertainty grows, higher transmission power is needed in order to guarantee users' QoS requirements. However, the results reveal that the proposed approach is effective in terms of QoS guarantees when the estimation error tends to increase more gradually. Therefore, the channel estimation method is practical in the fast fading environment where the channels change quickly and the users' QoS requirement are needed to be guaranteed such as $5 \mathrm{G}$ communications.

We evaluate the performance of the network in terms of worst-case received SINRs under different error variances for the existing algorithms in Fig. 3. The figure explicitly demonstrates the impact of estimation error on the network throughput. It is evident that there exists a reasonable tradeoff between received date rate and estimation error. The lower estimation error leads to achieve higher data rate. As channel estimation error is increased, the network performance becomes worse and worse. As a result, the network performance is decreased with increased channel estimation error.

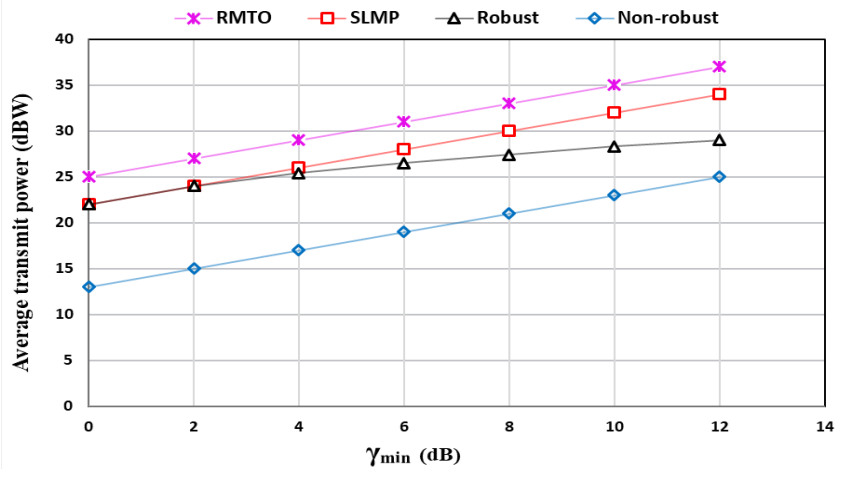

Fig. 2. Transmit power versus QoS guarantees $\left(\gamma_{\min }\right)$ for $\xi^{2}=0.01$ and $k=6$. 


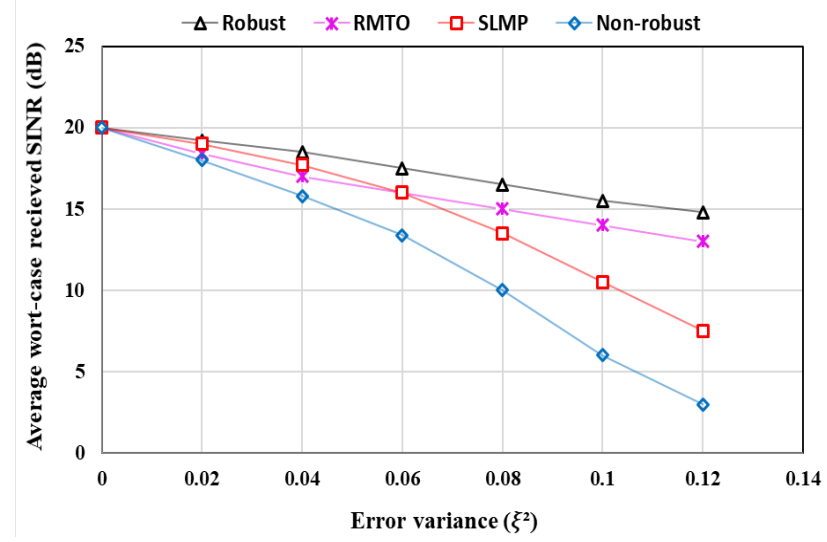

Fig. 3. Average worst-case received SINRs of the approaches for different $\xi^{2}$.

Fig. 4 Plots the average worst-case symbol error rates (SERs) of the algorithms under different SINRs. We calculate the SER as follows:

$$
\operatorname{MSE}\left(s_{k}\right)=\mathbb{E}\left\{\left|\hat{s}_{k}-s_{k}\right|^{2}\right\}
$$

Where $s_{k}$ is the vector of data symbols intended for the $k^{t h}$ user, and $\hat{s}_{k}=\left(f_{k}\right)^{-1} z_{k}$ states an estimate of the symbol intended for user $k$ so that $\mathbb{E}\left(s_{k} s_{k}^{H}\right)=I$.

We also investigated the impact of uncertainty size on the average worst-case SERs at SINR $=13 \mathrm{~dB}$ in Fig. 5. It can be clearly observed that the proposed method significantly outperforms the other algorithms and has the lowest worst-case SER under different uncertainty size.

As expected, both SLMP and RMTO schemes have higher SERs than the proposed approach, though consuming less power. That is because they only observe uncensored measurements, thus the state error covariance increases with each missed measurement. More precisely, the proposed method includes both fading and censored measurements which provides a more accurate estimation of the state that leads to lower SERs. That is to say, our method provides robustness against CSI errors compared to other methods even for a large uncertainty size or high SINR.

Although, there still exists a slight performance gap compared to the perfect CSI, however, the perfect CSI estimation is unrealistic in practice.

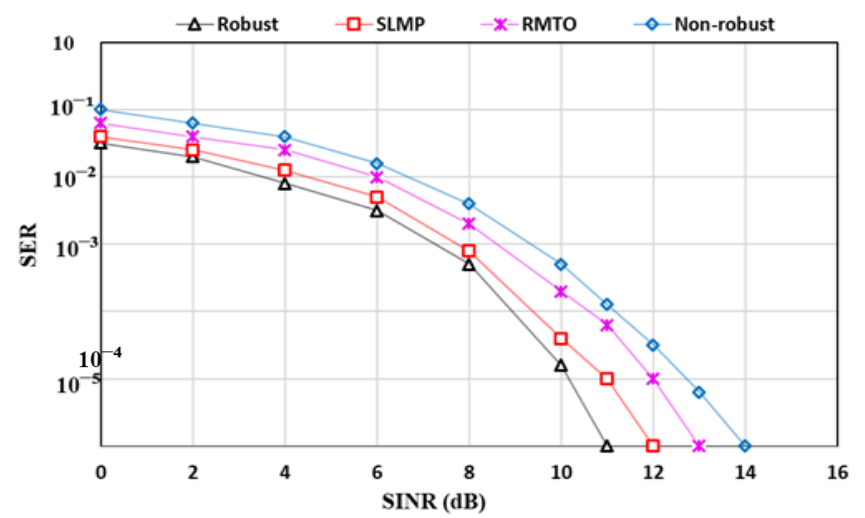

Fig. 4. Average worst-case users' SERs versus SINR for $\xi^{2}=0.001$ and $k=6$.

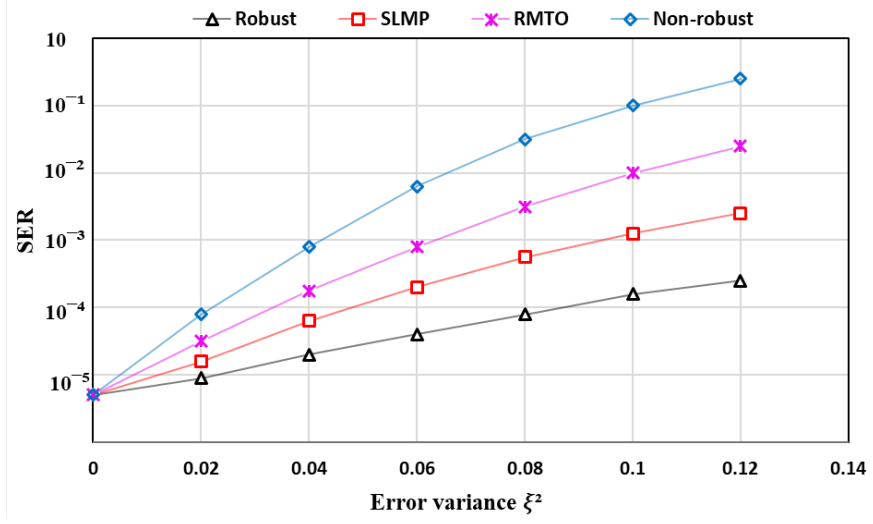

Fig. 5. Average worst-case users' SERs for different $\xi^{2}$ at $\mathrm{SINR}=13 \mathrm{~dB}$.

Fig. 6 shows the comparison of the average worst-case received SINR for the proposed approach and the existing schemes under different transmit power. As shown, the received SINR increases slowly in the non-robust schemes over other methods. Also, the received SINR of users grows when the transmit power of their serving BS increases. However, the increase in the received SINR for non-robust methods is much less than the robust algorithms since the received SINR depends on the accuracy of CSI. In fact, the non-robust methods are much more sensitive to imperfect CSI knowledge. Therefore, under worst channel uncertainty, the rise of estimation error decreases the received SINR for users in the non-robust designs. Moreover, there is a performance gap between the robust methods and the non-robust designs that verifies the superiority of the proposed algorithm in terms of robustness compared to the other methods under channel uncertainties. The gap gradually grows when the transmit power increases. This is due to the robustness of the proposed work against imperfect CSI.

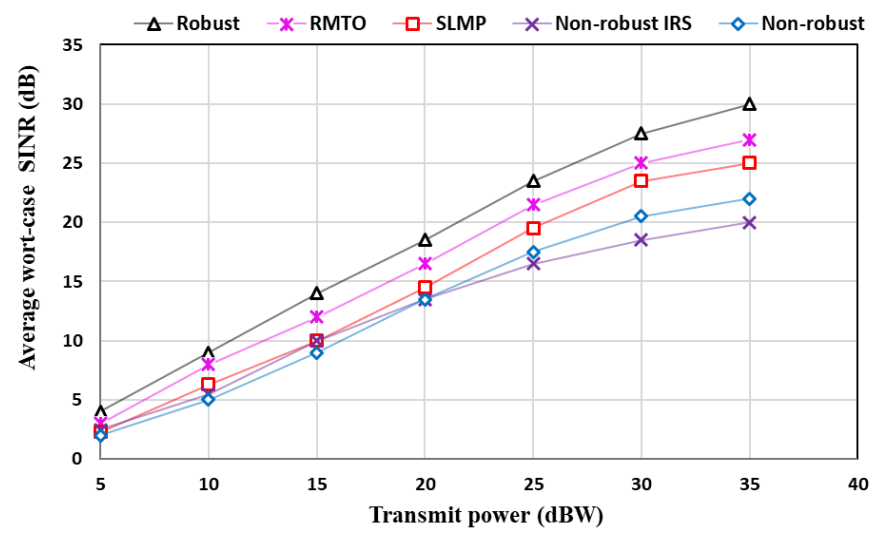

Fig. 6. Average worst-case received SINR under different transmit power

In addition, for more analysis, we have plotted the CDF of estimation error for the methods discussed in Fig.7. As presented in the graph, nearly $70 \%$ of the mean estimation error is below 0.3 in the proposed approach while this is $30 \%$ for the other schemes. The findings indicate that the proposed 
approach owns more accurate results and the lowest average estimation error.

$\triangle$-Robust $\square$-SLMP $-*$-RMTO $x$-Non-robust IRS $\diamond$-Non-robust

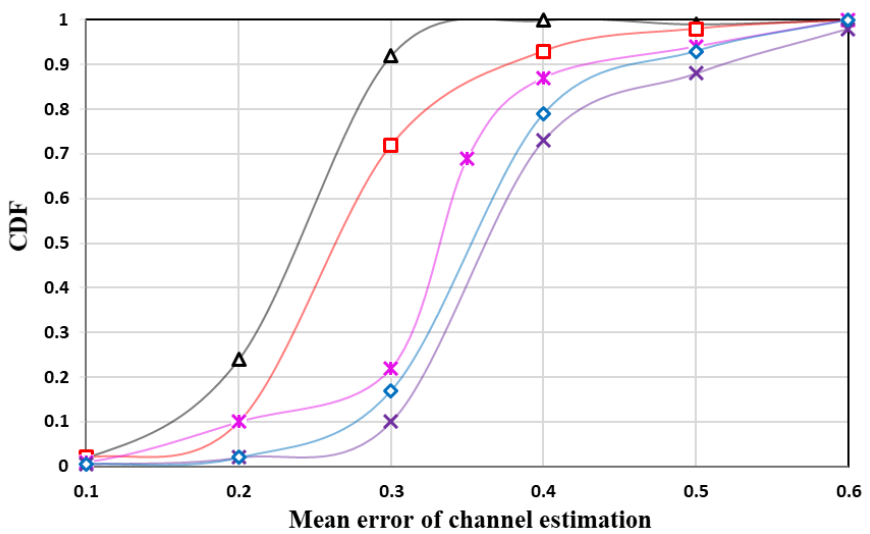

Fig. 7. The CDF of estimation error for $\xi^{2}=0.001$

Fig. 8 plots the impact of SINR values on the percentage of users with reliable SINR. The results show that the number of users with reliable SINR is increased in high SINR regions. In other words, when the SINR is increased, more users can meet their QoS requirement. In fact, the high value of SINR provides high data rate for the users and results in fulfilling users' QoS requirement. For instance, in regions with SINR $>10 \mathrm{~dB}$, at least $25 \%$ of users receive their required average data rate. This value reaches $45 \%$ when SINR goes beyond $25 \mathrm{~dB}$. However, a multiuser system needs high received SNR as well as good channel matrix condition [77]. Thus, obviously the average received SINR relies on the quality of channel matrix estimation. However, the number of users must be limited because the large number of users reduces channel capacity and the average received SINR for the users. This quantity serves as an upper bound on the SINR. For example, for SINR $>30 \mathrm{~dB}$, there would be no change on the percentage of users with reliable SINR.

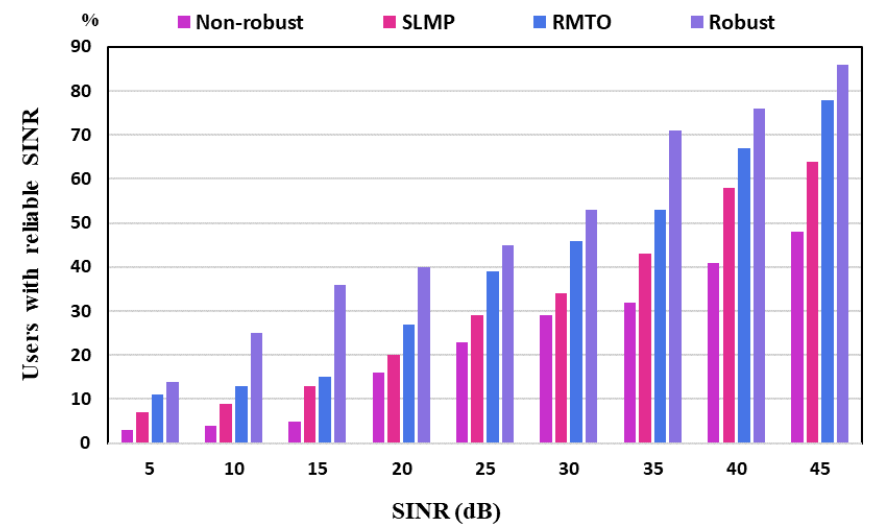

Fig. 8. The percentage of users with reliable SINR under different SINR

Fig. 9 depicts the convergence of the existing algorithms. The results prove that the proposed method converges to the MSE solution much faster than the other schemes. More precisely, our approach reaches stability during 12 iterations. However, its convergence speed depends on the initial values of the channel state.

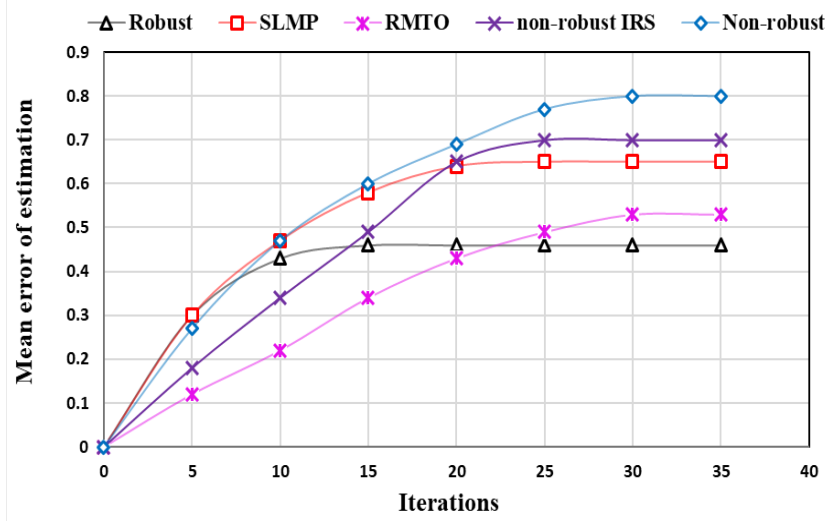

Fig. 9. The number of iterations of algorithm convergence for $B=25, K=45$ and $\xi^{2}=0.001$

Fig. 10 depicts spectral efficiency of the proposed approach under different base station densities. We have performed the experiments under different BS densities ranging from 5 to 25 while keeping the area of network deployment and the number of users unchanged. As shown in the Figure, the spectral efficiency monotonically grows with increasing the BS density. For example, when the number of BSs reaches 12, the spectral efficiency reaches $87 \%$ and when the spectral efficiency reduces to $71 \%$, there are only 9 BSs. However, the increasing number of the nearby interfering BSs decreases the capacity of system over time. For instance, when the spectral efficiency reaches $96 \%$ with 25 BSs, a greater number of BSs doesn't affect much. Therefore, compared to the existing approaches, the findings verify that the proposed method significantly enhances the spectral efficiency (i.e. 13\%).

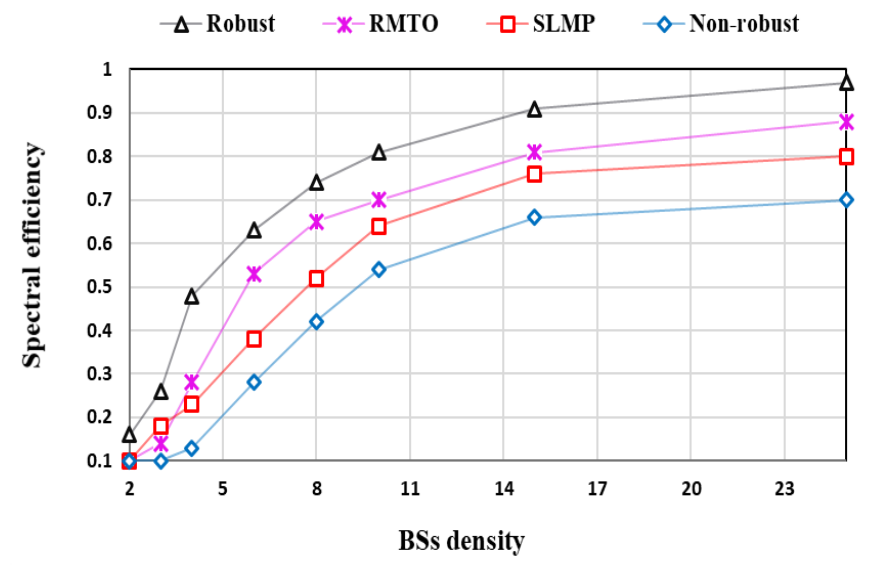

Fig. 10. Spectral efficiency under different base station densities for $\xi^{2}=$ 0.001 and $k=45$

In Fig. 11, we have also investigated the impact of the number of users on the spectral efficiency under fixed BSs density (i.e., with $18 \mathrm{BSs}$ ). As predicted, the growing number of users does not increase the spectral efficiency, because the network capacity degrades along with the greater number of users. 
$\square-$ SLMP $\triangle$-Robust $-\mathbb{X}$-RMTO $-\mathrm{X}$-non-robust IRS $\diamond$-Non-robust

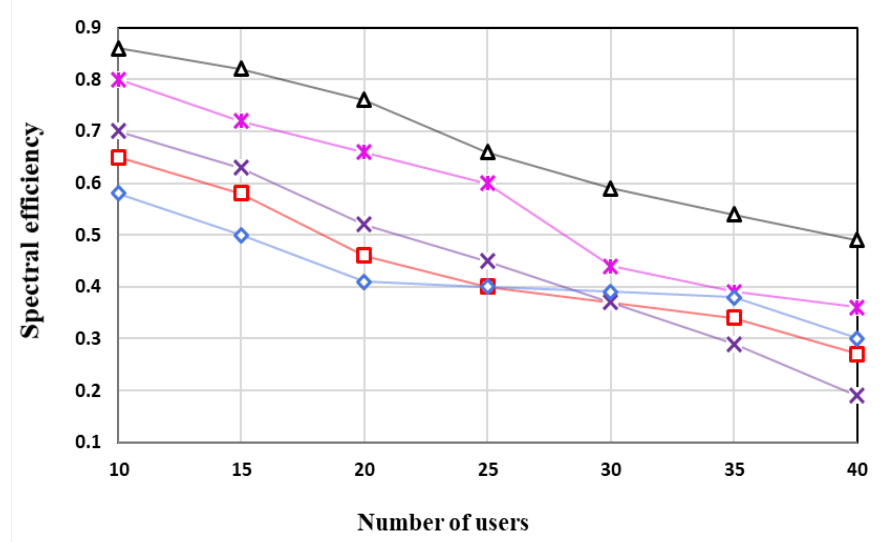

Fig. 11. Spectral efficiency under different number of users for $\xi^{2}=0.001$ and $B=18$

In Fig. 12, we investigate the impact of different number of users on the network performance in terms of average throughput for the three existing methods. We increased the number of wireless users up to 45 with 6 BSs and set the transmit power of BSs to $35 \mathrm{dBW}$. The experiments were conducted for the same given error variance $\xi^{2}=0.001$ for all the users and results were averaged over 80 independent runs. The results show that increasing number of wireless users increases the average throughput, too. The proposed approach can provide the average throughput up to $1 \mathrm{Mbps}$ as the proposed algorithm provides robustness against channel uncertainty compared to other methods. The accurate channel estimation significantly enhances the network capacity and improves the average throughput and network performance. However, the average throughput reaches a steady state and remains almost the same in our method while the simulation keeps up. This has been anticipated as increasing the number of interfering users decreases the capacity of network. With such an increase in the number of users, the total interference among wireless users grows significantly and users experience heavier interference, so that the average received SINR is reduced for the users. On the other hand, increasing the number of users reduces the channel capacity which results in decreasing throughput, too. Consequently, the proposed approach outperforms the other state-of-the-art schemes in terms of serving large numbers of users.

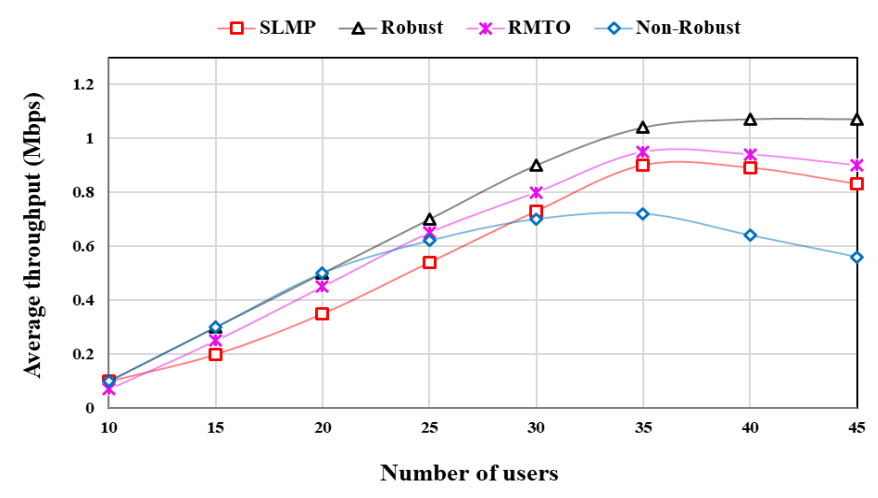

The energy efficiency of the approaches versus the antenna number under channel uncertainty has been analyzed and the superiority of the proposed method comparing to non-robust methods has been presented in Fig. 13. The energy efficiency, $E E$ can be defined as follows [79]:

$E E\left[\frac{\text { bits }}{\text { Joule }}\right]=\frac{\text { Data rate }\left(\frac{\text { bit }}{\text { s }}\right)}{\text { Energy consumption }}$

Therefore, we have

$$
E E=\frac{W \log _{2}\left(1+\frac{\left.P|h|\right|^{2}}{W N_{0}}\right)}{P}
$$

Where $W$ is a bandwidth hat the communication takes place over it, $P$ denotes the total transmit power of users that reflects energy consumption and $N_{0}$ states the noise power spectral density. Clearly, the energy efficiency is maximized when $P / W \rightarrow 0$ which is achieved by taking the transmit power $P \rightarrow$ 0 . We measured the energy efficiency of the proposed approach with 40 users for two different values of channel error variance: $\xi^{2}=0.001$ and $\xi^{2}=0.01$ and varied the number of antennas from 6 to 60 . It has been observed that the rate of power consumption with increasing the number of antennas in the proposed robust approach is considerably lower than the nonrobust schemes. When the number of antennas is increased, higher throughput is achieved. On the other hand, the proposed robust approach tracks the accurate channel states, thus the users adapt the transmit power to the channel gain and decrease their transmission power. As a result, power consumption in the network is reduced and energy efficiency (bits/Joule) is improved. The experiments also indicate that the degradation of energy efficiency degrades for larger error variance is higher. For instance, the proposed method achieves 4.3 bits/Joule at $N_{T}=18$ antennas under $\xi^{2}=0.01$ whereas it reaches 4.4 bits/Joule for $\xi^{2}=0.001$. However, the optimal energy efficiency with $N_{T}=18$ antennas under $\xi^{2}=0.001$ for the non-robust algorithm and the RMTO approach are 0.8 bits/Joule and $1.9 \mathrm{bits} / \mathrm{Joule}$, respectively.

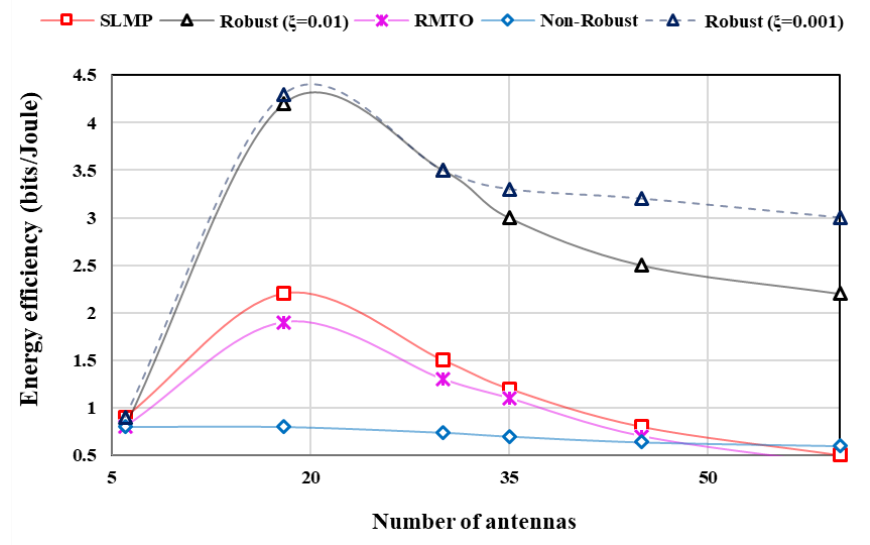

Fig. 13. Energy efficiency under different number of antennas for $\xi^{2}=0.001$ and $\xi^{2}=0.01, k=45$ and $\operatorname{SINR}=12 \mathrm{~dB}$

Fig. 12. The Average throughput under different number of users 
We have studied the battery drains of UEs in the existing algorithms in Fig. 14. The results confirm that the battery of the UEs in the proposed approach consumes less energy and have longer lifetime comparing to the other schemes. This is due to the effectiveness of the proposed distributed algorithm which reduces communication overhead and therefore, prolongs the battery life of the UEs. Moreover, increasing battery life of the UEs significantly improves energy efficiency of the network.

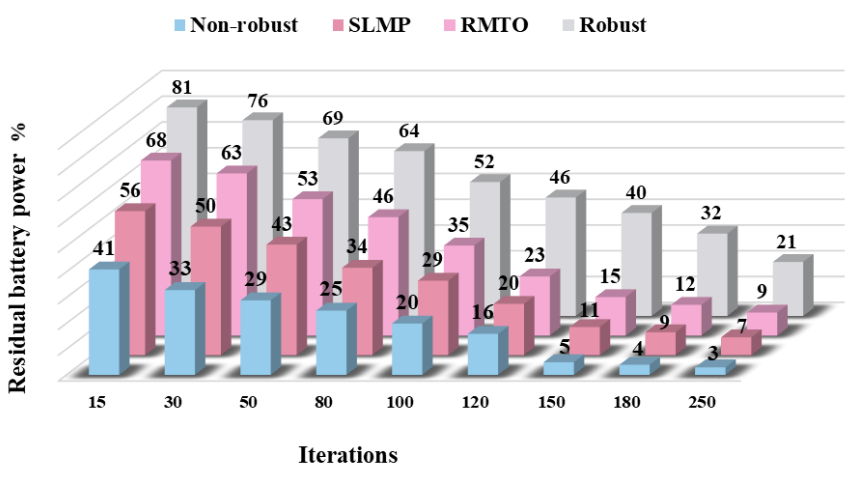

Fig. 14. The impact of radio communication on the battery drains of UEs.

\section{CONCLUSION}

In this paper, we developed a robust channel estimation approach for multiuser $5 \mathrm{G}$ communications with imperfect CSI. We transformed the downlink transmission to a discrete statespace model and established the Tobit Kalman filter to estimate the unknown state variables of a dynamic channel under uncertainty model. Unlike the existing works on the same topic, we investigated the censoring phenomenon by the Tobit measurement model to achieve more accurate estimation. The QoS requirements in terms of minimum SINR targets were taken into account and fulfilled for all the users. To minimize the maximum estimation error, we designed a robust minimax MSE estimation. Moreover, a game theoretic formulation was adopted to model the interactions among wireless users in a practical multiuser communication. The proposed game maximized the worst-case performance of each user under the worst uncertainty scenario. Theoretical analysis validates the robustness of the proposed method because of its insensitiveness to the uncertainty of the channel statistics. The algorithm can track the true values of the channel states through the expected values of the channel coefficient under the worst channel uncertainty. Furthermore, simulation results verified the effectiveness of the work in terms of both channel estimation accuracy and QoS guarantees for a wide uncertainty range.

\section{REFERENCES}

[1] A. Al-Fuqaha, M. Guizani, M. Mohammadi, M. Aledhari, and M. Ayyash., "Internet of Things: A survey on enabling technologies, protocols, and applications," IEEE Commun. Surveys Tuts., vol. 17, no. 4, pp. 2347-2376, 2015.

[2] M. R. Palattella, M. Dohler, A. Grieco, G. Rizzo, J. Torsner, T. Engel, and L. Ladid., "Internet of Things in the $5 \mathrm{G}$ era: Enablers, architecture, and business models," IEEE J. Sel. Areas Commun., vol. 34, no. 3, pp. $510-527,2016$.
[3] M.H. Miraz, M. Ali, P.S. Excell, and R. Picking., "A review on Internet of Things (IoT), Internet of everything (IoE) and Internet of nano things (IoNT)," In 2015 Internet Technologies and Applications (ITA), pp. 219224, 2015.

[4] P.P. Ray., "A survey on Internet of Things architectures," Journal of King Saud University-Computer and Information Sciences., vol. 30, no. 3, pp. 291-319, 2018.

[5] Nokia, "LTE evolution for IoT connectivity," Nokia, Espoo, Finland, White Paper, pp. 1-18, 2017.

[6] S. Li, L.Da. Xu, and S. Zhao., "5G Internet of Things: A survey,” Journal of Industrial Information Integration, vol. 10, pp.1-9, 2018.

[7] G.A. Akpakwu, B.J. Silva, G.P. Hancke, and M. A-M. Adnan., "A survey on $5 \mathrm{G}$ networks for the Internet of Things: Communication technologies and challenges," IEEE Access, vol. 6, pp. 3619-3647, 2017.

[8] M. Condoluci, G. Araniti, T. Mahmoodi, and M. Dohler., "Enabling the IoT machine age with 5G: Machine-type multicast services for innovative real-time applications," IEEE Access, vol. 4, pp. 5555-5569, 2016.

[9] A. Gupta and R.K. Jha., "A survey of 5G network: Architecture and emerging technology," IEEE Access, vol.3, pp. 1206-1232, 2015.

[10] Zhang, Q., Liu, F. and Zeng, C., 2019, April. Adaptive interferenceaware vnf placement for service-customized $5 \mathrm{~g}$ network slices. In IEEE INFOCOM 2019-IEEE Conference on Computer Communications (pp. 2449-2457). IEEE.

[11] C. Luo, J. Ji, Q. Wang, X. Chen, \& P. Li." Channel state information prediction for 5G wireless communications: A deep learning approach" IEEE Transactions on Network Science and Engineering, vol. 7, no 1, pp. 227-236, 2020.

[12] A. Ali, L. Feng, A. K. Bashir, S. H.A. El-Sappagh, S. H. Ahmed, M. Iqbal \& G. Raja. "Quality of service provisioning for heterogeneous

[13] services in cognitive radio-enabled Internet of Things" IEEE Transactions on Science and Engineering, vol. 7, no. 1, pp. 328-342, 2020.

[14] Y. L. Lee, T. C. Chuah, J. Loo, and A. Vinel, "Recent advances in radio resource management for heterogeneous LTE/LTE-A networks," IEEE Commun. Surveys Tutorials, vol. 16, no. 4, pp. 2142-2180, 2014.

[15] A. Pourkabirian, M. Dehghan Takht Fooladi, E. Zeinali, and A.M. Rahmani, "Base station selection in two-tier femtocell networks: A game-theoretic approach," Journal of New Researches in Mathematics. vol. 15, no. 1, pp. 167-178, 2019.

[16] J. Rao and A. Fapojuwo, "A survey of energy efficient resource management techniques for multicell cellular networks," IEEE Commun. Surveys Tutorials, vol. 16, no. 1, pp. 154-180, 2014.

[17] M. K. Awad, A. A. Behiry, \& E. A. Alrashed., "A Robust and Resilient Load Balancing Framework for SoftRAN-based HetNets with Hybrid Energy Supplies," IEEE Transactions on Network and Service Management, 2020.

[18] L. Lei, D. Yuan, C. K. Ho, and S. Sun, "Power and channel allocation for non-orthogonal multiple access in $5 \mathrm{G}$ systems: Tractability and computation," IEEE Trans. Wireless Commun., vol. 15, no. 12, pp. 8580-8594, 2016.

[19] D. Zhai, M. Sheng, X. Wang, Z. Sun, C. Xu, and J. Li, "Energy saving resource management for D2D and cellular coexisting networks enhanced by hybrid multiple access technologies," IEEE Transactions on Wireless Communications, vol. 16, no. 4, pp. 2678-2692, 2017.

[20] D. T. Ngo, S. Khakurel, and T. Le-Ngoc, "Joint sub-channel assignment and power allocation for OFDMA femtocell networks," IEEE Transactions on Wireless Communications, vol. 13, no. 1, pp. 342-355, 2014.

[21] M. F. Hanif, Z. Ding, T. Ratnarajah, and G. K. Karagiannidis, "A minorization-maximization method for optimizing sum rate in the downlink of non-orthogonal multiple access systems," IEEE Transactions on Signal Processing, vol. 64, no. 1, pp. 76-88, 2016.

[22] C. Papathanasiou, N. Dimitrio, and L. Tassiulas, "Dynamic radio resource and interference management for MIMO-OFDMA mobile broadband wireless access systems," Comput. Netw., vol. 57, no. 2013, pp. 3-16, 2013.

[23] A. C. Cagatay, R. Wang, Y. Hua, and M. Latva-aho, "Weighted sum rate maximization for full-duplex MIMO interference channels," IEEE Trans. Commun., vol. 63, no. 3, pp. 801-815, 2015.

[24] N. Mokari, F. Alavi, S. Parsaeefard, and T. Le-Ngoc, "Limited-feedback resource allocation in heterogeneous cellular networks," IEEE Transactions on Vehicular Technology, vol. 65, no. 4, pp. 2509-2521, 2016. 
[25] M. Moltafet, R. Joda, N. Mokari, M. R. Sabagh, and M. Zorzi, "Joint access and front haul radio resource allocation in PD-NOMA-based 5G networks enabling dual connectivity and CoMP," IEEE Transactions on Communications, vol. 66, no. 12, pp. 6463-6477, 2018.

[26] C. Yang, J. Li, Q. Ni, A. Anpalagan, and M. Guizani, "Interference aware energy efficiency maximization in 5G ultra-dense networks," IEEE Trans. Commun., vol. 65, no. 2, pp. 728-739, 2017.

[27] K. Wang, H. Li, F. R. Yu, and W. Wei, "Virtual resource allocation in software-defined information-centric cellular networks with device todevice communications and imperfect CSI," IEEE Transactions on Vehicular Technology, vol. 65, no. 12, pp. 10011-10021, 2016.

[28] K. Lee and C. Kim, "User scheduling for MU-MIMO transmission with active CSI feedback." EURASIP Journal on Wireless Communications and Networking, vol.1, 2015.

[29] M. A. Maddah-Ali and D. Tse, "Completely stale transmitter channel state information is still very useful," IEEE Transactions on Information Theory, vol. 58, no. 7, pp. 4418 - 4431, 2012.

[30] S. Mallick, R. Devarajan, R. A. Loodaricheh, and V. K. Bhargava, "Robust resource optimization for cooperative cognitive radio networks with imperfect CSI," IEEE Trans. Wireless Commun., vol. 14, no. 2, pp. 907-920, 2015.

[31] Y. Hu, Y. Xu, Y. Liu, and H. Yu, "Robust Energy-Efficiency Power Allocation in Multicell HetNets with Channel Uncertainties," In 2018 IEEE/CIC International Conference on Communications in China (ICCC), pp. 426-430. IEEE, 2018.

[32] Y. Xu, Y. Hu, and G. Li. "Robust rate maximization for heterogeneous wireless networks under channel uncertainties," Sensors, vol. 18, no. 2, 2018.

[33] M. Moltafet, S. Parsaeefard, M.R. Javan, and N. Mokari, "Robust Radio Resource Allocation in MISO-SCMA Assisted C-RAN in 5G Networks," IEEE Transactions on Vehicular Technology, vol. 6, no. 68, pp. 5758-5768, 2019.

[34] W. Yuan, N. Wu, Q. Guo, Y. Li, C. Xing, and J. Kuang, "Iterative receivers for downlink MIMO-SCMA: Message passing and distributed cooperative detection," IEEE Transactions on Wireless Communications, vol. 17, no. 5, pp. 3444-3458, 2018.

[35] A. Mokdad, M. Moltafet, P. Azmi, and N. Mokari, "Robust radio resource allocation for heterogeneous traffic in PD-NOMA-based HCNs," in Proc. IEEE Middle East and North Africa Communications Conference (MENACOMM), pp. 1-6, 2018.

[36] A.J. Muhammed, Z. Ma, P.D. Diamantoulakis, L. Li, and G.K. Karagiannidis, "Energy-Efficient Resource Allocation in Multicarrier NOMA Systems with Fairness," IEEE Transactions on Communications, vol. 12, no. 67, pp. 8639-8654, 2019.

[37] X. Song, L. Dong, J. Wang, L. Qin, and X. Han, "Energy Efficient Power Allocation for Downlink NOMA Heterogeneous Networks with Imperfect CSI," IEEE Access, vol. 7, pp. 39329-39340, 2019.

[38] X. Li, H. Ding, M. Pan, J. Wang, H. Zhang, and Y.Fang, "Statistical QoS provisioning over uncertain shared spectrums in cognitive IoT networks: A distributional robust data-driven approach," IEEE Transactions on Vehicular Technology, vol. 68, no. 12, pp. 12286-12300, 2019.

[39] S. Bhattarai, J. M. J. Park, B. Gao, K. Bian, and W. Lehr, "An overview of dynamic spectrum sharing: Ongoing initiatives challenges and a roadmap for future research," IEEE Trans. Cogn. Commun. Netw., vol. 2, no. 2, pp. 110-128, 2016.

[40] L. Duan, J. Huang, and B. Shou, "Investment and pricing with spectrum uncertainty: A cognitive operator's perspective," IEEE Trans. Mobile Comput., vol. 10, no. 11, pp. 1590-1604, 2011.

[41] A.C. Cirik, R. Wang, Y.Hua, and M. Latva-aho, "Weighted sum-rate maximization for full-duplex MIMO interference channels," IEEE Transactions on Communications, vol. 63, no. 3, pp. 801-815, 2015.

[42] Z. Jiang, A. Molisch, G. Caire, and Z. Niu, "Achievable rates of FDD massive MIMO systems with spatial channel correlation," IEEE Trans. Wireless Commun., vol. 14, pp. 2868-2882, 2015.

[43] Z. Jiang, Z. He, S. Chen, A. Molisch, S. Zhou, and Z. Niu, "Inferring remote channel state information: Cram'er-rao lower bound and deep learning implementation," in Proc. IEEE Global Commun. Conf. (GLOBECOM), 2018.

[44] D. Mishra and H. Johansson, "Optimal Least Squares Estimator and Precoder for Energy Beamforming Over IQ-Impaired Channels," IEEE Signal Processing Letters, vol. 26, no. 8, pp. 1207-1211, 2019.

[45] J.C. Estrada-Jiménez, B.G. Guzmán, M. J. F. G. Garcia, and V.P.G. Jimenez, "Superimposed Training-based Channel Estimation for MISO Optical-OFDM VLC," IEEE Transactions on Vehicular Technology, vol. 68 , no. 6, pp. 6161-6166, 2019.

[46] F.E. Asim, J. Nossek, F. Antreic, Ch. C. Cavalcante, and A. LF de Almeida. "Maximum Likelihood Channel Estimation for MillimeterWave MIMO Systems with Hybrid Beamforming," In WSA 2019; 23rd International ITG Workshop on Smart Antennas, pp. 1-6. VDE, 2019.

[47] E. Panayirci, M.T. Altabbaa, M. Uysal, and H.V. Poor, "Sparse Channel Estimation for OFDM-Based Underwater Acoustic Systems in Rician Fading with a New OMP-MAP Algorithm," IEEE Transactions on Signal Processing, vol. 67, no. 6, pp. 1550-1565, 2019.

[48] M. Zhou, Z. Feng, and X. Huang, "Maximum A Posteriori Probability (MAP) Joint Fine Frequency Offset and Channel Estimation for MIMO Systems with Channels of Arbitrary Correlation," arXiv preprint arXiv:1905.03808, 2019.

[49] M. Martone, "Optimally regularized channel tracking techniques for sequence estimation based on cross-validated subspace signal processing," IEEE Trans. Commun, vol. 48, no. 1, pp. 95- 105, 2000.

[50] J. Rodriguez-Fernandez, N. Gonzalez-Prelcic, and T.Shimizu, "Channel Tracking and Hybrid Precoding for Wideband Hybrid Millimeter Wave MIMO Systems," arXiv preprint arXiv:1905.03903, 2019.

[51] O. Mehanna and N. D. Sidiropoulos, "Channel tracking and transmit beamforming with frugal feedback," IEEE Trans. Signal Process., vol. 62, no. 24, pp. 6402-6413, 2014.

[52] S. Kar, B. Sinopoli, and J.M.F Moura, "Kalman filtering with intermittent observations: Weak convergence to a stationary distribution," IEEE Transactions on Automatic Control, vol. 57, no. 2, pp. 405-420, 2012.

[53] C. Miller, M.J. Piovoso, R. Zurakowski, and Bethany Allik, "The tobit kalman filter: An estimator for censored data," IEEE Transactions on Control Systems Technology, vol. 24, no. 1, pp. 365-371, 2015.

[54] H Geng, Z Wang, Y Liang, Y Cheng, and FE Alsaadi, "Tobit Kalman filter with fading measurements," Signal Processing, vol. 1, no. 140, pp.60-68, 2017.

[55] K. Loumponias, N. Vretos, G. Tsaklidis, and P. Daras, "An Improved Tobit Kalman Filter with Adaptive Censoring Limits," arXiv preprint arXiv:1911.06190, 2019.

[56] Xiong, J., Zhan, Y., Yang, N. and Yang, L., 2020, October. Laser Spot Centroid Locating and Tracking Based on Tobit Kalman Filter in FSO Communications. In Asia Communications and Photonics Conference (pp. M4A-301). Optical Society of America.

[57] Chiariotti, F., 2019. The Adaptive Tobit Kalman Filter: Tracking Position with Censored Measurements in the IoT. arXiv preprint arXiv:1909.05603.

[58] S. Lasaulce and H. Tembine, "Game theory and learning for wireless networks: fundamentals and applications"” Academic Press, 2011.

[59] A. Pourkabirian, M. Dehghan Takht Fooladi, E. Zeinali, and A.M. Rahmani, "An Evolutionary Game-Theoretic Approach for Base Station Allocation in Wireless Femtocell Networks," Wireless Personal Communications, vol. 107, no. 1, pp. 217-242, 2019.

[60] A. Pourkabirian, M. Dehghan Takht Fooladi, E. Zeinali, and A.M. Rahmani, "Dynamic resource allocation for OFDMA femtocell networks: a game-theoretic approach," Telecommunication Systems, vol. 69, no. 1, pp. 51-59, 2018.

[61] K. Zhu, E. Hossain, and A. Anpalagan, "Downlink power control in twotier cellular OFDMA networks under uncertainties: A robust Stackelberg game," IEEE Trans. Commun., vol. 63, no. 2, pp. 520-535, 2015.

[62] H. Zhang, C. Jiang, N.C. Beaulieu, X. Chu, X. Wang, and T.Q.S. Quek, "Resource allocation for cognitive small cell networks: A cooperative bargaining game theoretic approach," IEEE Transactions on Wireless Communications, vol. 14, no. 6, pp. 3481-3493, 2015.

[63] P. Semasinghe, E. Hossain, and K. Zhu, "An evolutionary game for distributed resource allocation in self-organizing small cells," IEEE Transactions on Mobile Computing, vol. 14, no. 2, pp. 274-287, 2015.

[64] K. Zhu, E. Hossain, and D. Niyato, "Pricing, spectrum sharing, and service selection in two-tier small cell networks: A hierarchical dynamic game approach," IEEE Transactions on Mobile Computing, vol. 13, no. 8, pp. 1843-1856, 2014.

[65] Z. Feng, L. Song, Z. Han, and X. Zhao, "Cell selection in two-tier femtocell networks with open/closed access using evolutionary game," In Wireless Communications and Networking Conference (WCNC) IEEE, pp. 860-865, 2013.

[66] P. Zhu, J. Bao, L. Zhang, and J. Li, "A pilot allocation algorithm based on coalitional game theory for distributed MIMO systems," IEEE Access, 7, pp.105996-106001, 2019.

[67] Z. Wang, F. Yang, S. Yan, S. Memon, Z. Zhao, and C. Hu, , “ Joint 
design of coalition formation and semi-blind channel estimation in fog radio access networks," China Communications, vol. 16, no. 11, pp.115, 2019.

[68] B. Allik, C. Miller, M.J. Piovoso, and R. Zurakowski, "Estimation of saturated data using the Tobit Kalman filter," In 2014 American Control Conference, IEEE, pp. 4151-4156, 2014.

[69] B. Allik, C. Miller, M.J. Piovoso, and R. Zurakowski, "The Tobit Kalman filter: an estimator for censored measurement," IEEE Transactions on Control Systems Technology, vol. 24, no. 1, pp. 365371,2015

[70] H. Dong, Z. Wang, S.X. Ding, and H. Gao, "On H-infinity estimation of randomly occurring faults for a class of nonlinear time-varying systems with fading channels," IEEE Transactions on Automatic Control, vol. 61, no. 2, pp. 479-484, 2015.

[71] X. Zhang," Matrix Analysis and Applications," Tsinghua University Press, 2004

[72] D. Coppersmith and S. Winograd, "On the asymptotic complexity of matrix multiplication," SIAM Journal on Computing, vol. 11, no. 3, pp. 472-492, 1982

[73] A. Haqiqatnejad, F. Kayhan, and B. Ottersten, "Robust SINRConstrained Symbol-Level Multiuser Precoding with Imperfect Channel Knowledge," IEEE Transactions on Signal Processing, vol. 1, no. 68, pp. 1837-1852, 2020.

[74] M. Shao and W.K. Ma, "A simple way to approximate average robust multiuser MISO transmit optimization under covariance-based CSIT," In 2017 IEEE International Conference on Acoustics, Speech and Signal Processing (ICASSP), pp. 3504-3508. IEEE, 2017.

[75] Zhang, J., Zhang, Y., Zhong, C. and Zhang, Z., 2020. Robust design for intelligent reflecting surfaces assisted MISO systems. IEEE Communications Letters, 24(10), pp.2353-2357.

[76] (2018, Dec.) CVX: MATLAB software for disciplined convex programming. [Online]. Available: http://cvxr.com/cvx

[77] N. Vucic and H. Boche, "Robust QoS-constrained optimization of downlink multiuser MISO systems," IEEE Transactions on Signal Processing, vol. 57, no. 2, pp. 714-725, 2008.

[78] Sampath, H., Talwar, S., Tellado, J., Erceg, V. and Paulraj, A., 2002. A fourth-generation MIMO-OFDM broadband wireless system: design, performance, and field trial results. IEEE Communications Magazine, 40(9), pp.143-149.

[79] Björnson, Emil, and Erik G. Larsson. "How energy-efficient can a wireless communication system become?." In 2018 52nd Asilomar Conference on Signals, Systems, and Computers, pp. 1252-1256. IEEE, 2018.

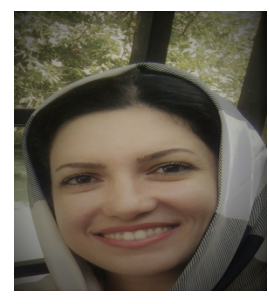

Azadeh Pourkabirian received the M.Sc. degree in Computer Network from Qazvin Azad University, Iran, in 2007, and the Ph.D. degree in Computer Science from Science and Research Branch of Azad University, Iran, in 2018. She is currently an Associate Professor with the Department of Computer Science and Information Technology, Qazvin Azad University. Her research interests include wireless communication, $5 \mathrm{G}$, resource allocation, optimization, stochastic modeling, and game theory.

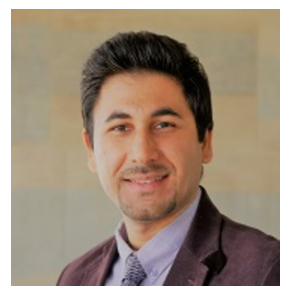

Mohammad Hossein Anisi is an Associate Professor at the School of Computer Science and Electronic Engineering, University of Essex, UK and head of Internet of Everything Laboratory. Prior to that, he worked as a Senior Research Associate at University of East Anglia, UK and Senior Lecturer at University of Malaya, Malaysia where he received 'Excellent Service Award' for his achievements. His research has focused specifically on real world application domains such as energy management, transportation, healthcare and other potential life domains. He has designed and developed novel Internet of Things (IoT)-based communication systems and his research results have directly contributed to the technology industry. He has strong collaboration with industry and working with several companies in the UK with the focus on monitoring and automation systems based on IoT concept capable of reliable and seamless generation, transmission, processing and demonstration of data. He has published more than 100 articles in high quality journals and several conference papers and won two medals for his innovations from PECIPTA 2015 and IIDEX 2016 expositions. He has received several International and national funding awards for his fundamental and practical research as PI and Co-I. Dr Anisi is an associate editor of a number of journals including 'IEEE Access', 'Ad Hoc Sensor Wireless Networks', 'IET Wireless Sensor Systems', 'International Journal of Distributed Sensor Networks'. 'KSII Transactions on Internet and Information Systems journals' and 'Journal of Sensor and Actuator Networks'. He has been Lead organizer of special sessions and workshops at IEEE conferences such as IEEE ICC, IEEE CAMAD, IEEE PIMRC, IEEE VTC and etc. He has been also serving as executive/technical committee member of several conferences. Hossein is Fellow of Higher Education Academy and Senior Member of IEEE. He is also a technical committee member of Finnish-Russian University Cooperation in Telecommunications (FRUCT), Senior Member of Institute of Research Engineers and Doctors (the IRED), Member of ACM, IEEE Council on RFID, IEEE Sensors Council, IEEE Systems Council and International Association of Engineers (IAENG). 